\title{
A THEOREM OF GRACE ON THE ZEROS OF POLYNOMIALS, REVISITED
}

\author{
J. L. WALSH ${ }^{1}$
}

A theorem of Grace [1] has shown itself highly useful (e.g. [2]) in the study of the geometry of the zeros of polynomials. We shall treat not that theorem in full generality, but the following special case:

LEMмA 1. If we have $m_{k}>0, \sum m_{k}=1,\left|\alpha_{k}\right| \leqq 1, k=1,2, \cdots, n$, $|z|>1$, then the equation in $\alpha$

$$
\prod_{k=1}^{n}\left(z-\alpha_{k}\right)^{m_{k}}=z-\alpha
$$

has a solution $\alpha$ which satisfies $|\alpha| \leqq 1$. Indeed there exists such a solution $\alpha$ satisfying

$$
\min _{z} \arg \left[\left(z-\alpha_{k}\right) / z\right] \leqq \arg [(z-\alpha) / z] \leqq \max _{k} \arg \left[\left(z-\alpha_{k}\right) / z\right]
$$

where these three arguments are values of any $\arg [(z-\beta) / z]$ chosen continuous for fixed $z$ and for all $\beta$ with $|\beta| \leqq 1$.

The latter part of Lemma 1 is unusual, but is to be established below. Lemma 1 is valid [2, Theorem III] without the hypothesis $|z|>1$ if (2) is omitted.

If the $m_{k}$ and $\alpha_{k}$ are fixed in Lemma 1 and $|z|$ is large, the point $\alpha$ (which depends on $z$, with $|\alpha| \leqq 1$ ) lies near the center of gravity of the $\alpha_{k}$, as we see by writing (1) in the form

$$
\begin{aligned}
& \sum m_{k} \log \left(1-\frac{\alpha_{k}}{z}\right) \\
& \equiv \sum m_{k}\left[-\frac{\alpha_{k}}{z}-\frac{1}{2}\left(\frac{\alpha_{k}}{z}\right)^{2}-\frac{1}{3}\left(\frac{\alpha_{k}}{z}\right)^{3}-\cdots\right] \\
& \equiv \log \left(1-\frac{\alpha}{z}\right) \equiv\left[-\frac{\alpha}{z}-\frac{1}{2}\left(\frac{\alpha}{z}\right)^{2}-\frac{1}{3}\left(\frac{\alpha}{z}\right)^{3}-\cdots\right] ;
\end{aligned}
$$

yet this important fact is not mentioned in Lemma 1. The purpose of this paper is to prove, and to apply in illustrative cases, a revision of Lemma 1:

Received by the editors October 1, 1962 and, in revised form, February 2, 1963.

1 This research was supported (in part) by the Air Force Office of Scientific Research. Abstract in Notices Amer. Math. Soc. 9 (1962), 484. 
Lemma 2. If we have $m_{k}>0, \sum m_{k}=1,\left|\alpha_{k}\right| \leqq 1, \sum m_{k} \alpha_{k}=0,|z|>1$ (where $k=1,2,3, \cdots, n$ ) then there exists an $\alpha$ such that $|\alpha| \leqq 1 /|z|$, with

$$
\sum m_{k} \log \left(1-\frac{\alpha_{k}}{z}\right)=\log \left(1-\frac{\alpha}{z}\right),
$$

where $\arg (1-\alpha / z)$ may be chosen as in (2).

Precisely the remark already made, that for large $|z|$ the point $\alpha$ lies near the center of gravity of the $\alpha_{k}$, applies also to another lemma which is of frequent use in the study of the geometry of zeros of polynomials, namely

LEMмA 3. If we have $m_{k}>0$. $\sum m_{k}=1,\left|\alpha_{k}\right| \leqq 1, k=1,2, \cdots, n$, $|z|>1$, then $\alpha$ as defined by the :quation

$$
\sum_{k=1}^{n} \frac{m_{k}}{z-\alpha_{k}}=\frac{1}{z-\alpha}
$$

satisfies $|\alpha| \leqq 1$.

The analogue of (3) is here the equivalent of (5):

$$
\begin{aligned}
\sum \frac{m_{k}}{z-\alpha_{k}} & \equiv \sum \frac{m_{k}}{z}\left[1+\frac{\alpha_{k}}{z}+\left(\frac{\alpha_{k}}{z}\right)^{2}+\cdots\right] \\
& \equiv \frac{1}{z-\alpha} \equiv \frac{1}{z}\left[1+\frac{\alpha}{z}+\left(\frac{\alpha}{z}\right)^{2}+\cdots\right]
\end{aligned}
$$

As the author has recently indicated [4], there exists a modification of Lemma 3 which bears precisely the same relation to Lemma 3 that Lemma 2 bears to Lemma 1:

LEMмA 4. Under the conditions of Lemma 3 and with $\sum m_{k} \alpha_{k}=0$, we have $|\alpha| \leqq 1 /|z|$.

Before proceeding with the proof of Lemma 2 we remark that the unit disk $|\zeta|<1$ is mapped conformally onto a convex region $R$ of the $w$-plane by the transformation $w=\log (1-\zeta)$. It follows that the image $R_{r}$ in the $w$-plane of the disk $|\zeta| \leqq r(<1)$ is also convex.

Equation (4), which defines $\alpha$, expresses $\log (1-\alpha / z)$ in the $w-$ plane as the center of gravity of the weighted points $w=\log \left(1-\alpha_{k} / z\right)$ of $R$. To prove Lemma 1 it is sufficient to choose $z=1 / r$, where $r$ is less than unity. Here we have $\left|\alpha_{k} / z\right| \leqq r$, so all points $\log \left(1-\alpha_{k} / z\right)$ lie in $R_{r}$ as does their center of gravity. That is, $\log (1-\alpha / z)$ lies in $R_{r}$ and (to return to the $\zeta$-plane) $\zeta=\alpha / z$ satisfies $|\alpha / z| \leqq r$, whence $|\alpha| \leqq 1$. 
The last part of Lemma 1 follows at once, so Lemma 1 is established.

The proof of Lemma 1 as just given has not been previously published in detail; this proof was outlined in [2, see Theorem III]. We proceed with the proof of Lemma 2. For $|z|>1$ we set $z^{\prime}=1 / z$ with $\left|z^{\prime}\right|<1$, and define $\alpha=\alpha\left(z^{\prime}\right)$ by equation (4) in the form

$$
\sum m_{k} \log \left(1-\alpha_{k} z^{\prime}\right)=\log \left(1-\alpha z^{\prime}\right) \text {. }
$$

We choose $\alpha\left(z^{\prime}\right)$ as in the proof of Lemma 1, and it follows from Lemma 1 that we have $\left|\alpha\left(z^{\prime}\right)\right| \leqq 1$. By the proof of Lemma 1 we may write

$$
\begin{aligned}
\alpha\left(z^{\prime}\right) & \equiv\left[1-\Pi\left(1-\alpha_{k} z^{\prime}\right)^{m_{k}}\right] / z^{\prime} \\
& \equiv\left[1-\Pi\left(1-m_{k} \alpha_{k} z^{\prime}+\frac{m_{k}\left(m_{k}-1\right)}{2 !} \alpha_{k}^{2} z^{\prime 2}-\cdots\right)\right] / z^{\prime}, \\
\alpha(0) & =0 .
\end{aligned}
$$

Schwarz's lemma applied to $\alpha\left(z^{\prime}\right)$ now yields

$$
|\alpha| \leqq\left|z^{\prime}\right|=1 /|z|,
$$

so Lemma 2 is established.

We remark that if also $\sum m_{k} \alpha_{\mathbf{k}}^{2}=0$, then we have $\alpha^{\prime}(0)-0$, and there follows $|\alpha| \leqq 1 /|z|^{2}$. A similar remark follows if we have $\alpha^{\prime \prime}(0)=0$, etc.

It is to be mentioned that there exists no number $\lambda(<1)$ such that for all $z$ with $|z|>1$ inequality (7) can be replaced by

$$
|\alpha| \leqq \lambda /|z|
$$

with the hypothesis of Lemma 2 . Indeed we choose $z>1, n=2$, $m_{1}=m_{2}, \alpha_{1}=1, \alpha_{2}=-1$, so there follows

$$
\begin{aligned}
(z-1)(z+1) & =z^{2}-1=(z-\alpha)^{2} \\
\alpha & =z-\sqrt{ }\left(z^{2}-1\right) .
\end{aligned}
$$

When $z \rightarrow 1$, we have $\alpha \rightarrow 1$, which contradicts (8).

In the special case just considered except that $\alpha_{1}=-\alpha_{2},\left|\alpha_{1}\right|=1$, and $|z|>1$, we have

$$
\begin{aligned}
\left(z-\alpha_{1}\right)\left(z+\alpha_{1}\right) & =(z-\alpha)^{2}, \quad \alpha_{1}^{2}=\alpha(2 z-\alpha), \\
|\alpha(2 z-\alpha)| & =1 .
\end{aligned}
$$

It follows that the locus of $\alpha$ is one oval of lemniscate (9), and we have $\max |\alpha|=|z|-\left(|z|^{2}-1\right)^{1 / 2}, \min |\alpha|=\left(|z|^{2}+1\right)^{1 / 2}-|z|$. If we require merely $\alpha_{1}=-\alpha_{2},\left|\alpha_{1}\right| \leqq 1$, the locus of $\alpha$ is the closed interior of the same oval of (9). 
The proof of Lemma 4, announced in [3] and hitherto unpublished, is primarily geometric in character and relatively involved. The comparatively simple proof published in [4] results from later suggestions made to the present writer by Mr. S. Jacobs, Professor L. Carleson, and Dr. Z. Rubinstein. The present proof of Lemma 2 is quite similar to the latter proof [4] of Lemma 4.

Lemmas 1 and 3 are the special cases $f(\zeta) \equiv \log (1-\zeta)$ and $f(\zeta)$ $\equiv 1 /(1-\zeta)$ of the following proposition: Let $w=f(\zeta)$ be schlicht-convex in $|\zeta|<1$, hence also in $|\zeta|<r(<1)$. Let $z$ be fixed, $|z|>1$, and suppose $\left|\alpha_{k}\right| \leqq 1, m_{k}>0, \sum_{1}^{n} m_{k}=1$. Then the equation in $\alpha$

$$
\sum_{k=1}^{n} m_{k} f\left(\frac{\alpha_{k}}{z}\right)=f\left(\frac{\alpha}{z}\right)
$$

has a solution $\alpha=\alpha(z)$ with $|\alpha| \leqq 1$. This solution can be restricted so as to be unique. Lemmas 2 and 4 are special cases of the corollary: If $\sum m_{k} \alpha_{k}=0$, then $\alpha(\infty)=0$, and we have $|\alpha(z)| \leqq 1 /|z|$ for $|z|>1$.

Lemmas 2 and 4 have important differences. The latter determines in fact for each even $n$ and for every $z$ with $|z|>1$ the actual locus of the point $\alpha$, but Lemma 2 does not. Moreover each holds for $|z|>1$ and not for $|z|<1$. This is not serious in Lemma 4 , for if $n>3$ each point of $|z|<1$ is a possible multiple point $\alpha_{k}$ and is ordinarily a point of the locus of zeros of the polynomial studied, but not so in the applications of Lemma 2 , where the point set $|z| \leqq 1$ requires separate and independent study, perhaps with the aid of the (valid) first part of Lemma 1 , but involving now $|z| \leqq 1$ and without the conclusion concerning arg $(1-\alpha / z)$.

Inequality (7) is not sharp, and we indicate rapidly a slight improvement. Let us set $\phi\left(z^{\prime}\right) \equiv \sum m_{k} \log \left(1-\alpha_{k}-z^{\prime}\right), \psi\left(z^{\prime}\right) \equiv 1$ $-\exp \left[\phi\left(z^{\prime}\right)\right], z^{\prime}=1 / z, \psi^{\prime \prime}(0)=-\phi^{\prime \prime}(0)=\sum m_{k} \alpha_{k}^{2}=b$, whence $|b| \leqq 1$. We set also $\Psi\left(z^{\prime}\right) \equiv \psi\left(z^{\prime}\right)-b z^{\prime 2} / 2$, whence $\Psi(0)=\Psi^{\prime}(0)=\Psi^{\prime \prime}(0)=0$, and for $\left|z^{\prime}\right|<1$ we have $\left|\Psi\left(z^{\prime}\right)\right|<1+|b| / 2$. Schwarz's lemma then yields, for $\left|z^{\prime}\right|<1$,

$$
\begin{aligned}
\left|\psi\left(z^{\prime}\right)-\frac{b z^{\prime 2}}{2}\right| & \leqq\left(1+\frac{|b|}{2}\right)\left|z^{\prime}\right|^{3} \\
\left|\psi\left(z^{\prime}\right)\right| & =\left|\alpha z^{\prime}\right| \leqq \frac{\left|b z^{\prime 2}\right|}{2}+\left(1+\frac{|b|}{2}\right)\left|z^{\prime}\right|^{3} \\
|\alpha| & \leqq\left|z^{\prime}\right|^{2}+\frac{|b|}{2}\left(\left|z^{\prime}\right|+\left|z^{\prime}\right|^{2}\right)
\end{aligned}
$$

when $z^{\prime}=0$ this inequality still holds, with $\alpha=0$, so we have 


$$
|\alpha| \leqq \frac{1}{|z|^{2}}+\frac{|b|}{2}\left(\frac{1}{|z|}+\frac{1}{|z|^{2}}\right) \leqq \frac{1}{2|z|}+\frac{3}{2|z|^{2}} .
$$

The extreme members of (10) give a stronger inequality than (7) whenever we have $|z|>3$.

We turn now to some relatively immediate applications of Lemma 2.

Theorem 1. Suppose $\left|\alpha_{k}\right| \leqq 1$ for $k=1,2, \cdots, n$, with $\sum \alpha_{k}=0$; we set $p(z) \equiv \prod\left(z-\alpha_{k}\right)-C$, where the constant $C$ is arbitrary. Then for $|z| \leqq 1$ all zeros of $p(z)$ lie in the $n$ circles $\left|z-C^{1 / n}\right| \leqq 1$ and for $|z|>1$ in the $n$ lemniscate regions

$$
\left|z\left(z-C^{1 / n}\right)\right| \leqq 1
$$

where $C^{1 / n}$ takes all $n$ values.

For $|z| \leqq 1$ we apply the analogue [2, Theorem III] of Lemma 1 involving merely $|\alpha| \leqq 1$; if $p(z)=0$ we have

$$
(z-\alpha)^{n}-C=0,
$$

whence $\alpha=z-C^{1 / n}$, so the first part of the conclusion follows. For $|z|>1$ we apply Lemma 2 , to obtain $|\alpha| \leqq 1 /|z|$,

$$
\left|z-C^{1 / n}\right| \leqq 1 /|z|
$$

and (11) follows. Thus all zeros of $p(z)$ lie in the parts (if any) of the $n$ discs $\left|z-C^{1 / n}\right| \leqq 1$ contained in the closed unit disc plus the parts (if any) of the regions (11) contained in the exterior of the unit disc. Any intersection of the set (11) with the unit circumference lies in one of the $n$ discs, and conversely, any intersection of one of the $n$ discs with the unit circumference lies in one of the sets (11), so the boundaries of the $n$ discs join continuously with the boundaries of the respective $n$ lemniscate regions. However, if we have $|C|>2^{n}$, the $n$ discs lie exterior to the unit disc; all zeros of $p(z)$ lie in the $n$ regions consisting each of the closed interior of that oval of a lemniscate (11) containing $C^{1 / n}$. It is readily shown by the method of continuity that if these latter $n$ regions are mutually disjoint, each contains precisely one zero of $p(z)$. Even if we have $1<|C|<2^{n}$, it may occur that the point set already described as containing all zeros of $p(z)$ falls into $n$ mutually disjoint closed regions, each bounded in part by an arc in $|z| \leqq 1$ of a circle $\left|z-C^{1 / n}\right|$ and in part by an arc in $|z|>1$ of the corresponding lemniscate; in this case, too, each such closed region contains precisely one zero of $p(z)$.

It may be noted that if $|C|<2^{n}$ the lemniscate $\left|z\left(z-C^{1 / n}\right)\right|=1$ consists of a single Jordan curve, whereas if $|C|>2^{n}$ it consists of 
two mutually exterior ovals contained in the respective closed discs whose centers are zero and $C^{1 / n}$, having the common radius $\left[C^{1 / n}-\left(C^{2 / n}-4\right)^{1 / 2}\right] / 2$, a radius less than unity. This radius approaches zero as $\left|C^{1 / n}\right|$ becomes infinite, in great contrast to the corresponding locus [2, Theorem V] yielded by application of the first part of Lemma 1 but for $|z|>1$, which consists of discs whose centers are the points $C^{1 / n}$ and which have the common radius unity.

We state another application of Lemma 2, related to Theorem IV of [2].

Theorem 2. Suppose we have $\left|\alpha_{k}-a\right| \leqq r_{1}$ and $\left|\beta_{k}-b\right| \leqq r_{2}, k=1,2$, $\cdots, n$, with $\sum \alpha_{k}=n a, \sum \beta_{k}=n b$. We set $p(z) \equiv \prod\left(z-\alpha_{k}\right)$ $-A \prod\left(z-\beta_{k}\right)$, where $A$ is an arbitrary constant. Then if $A \neq 1$ all zeros of $p(z)$ lie in the $n$ loci

$$
\begin{aligned}
\left|z-\frac{a-b A^{1 / n}}{1-A^{1 / n}}\right| \leqq\left|1-A^{1 / n}\right|^{-1} & {\left[r_{1} \cdot \min \left(1, \frac{r_{1}}{|z-a|}\right)\right.} \\
& \left.+r_{2}|A|^{1 / n} \cdot \min \left(1, \frac{r_{2}}{|z-b|}\right)\right]
\end{aligned}
$$

where $A^{1 / n}$ is in turn each nth root of $A$.

If $A=1$ and we have

$$
r_{1} \cdot \min \left(1, \frac{r_{1}}{|z-a|}\right)+r_{2} \cdot \min \left(1, \frac{r_{2}}{|z-b|}\right)>|a-b|,
$$

then all zeros of $p(z)$ lie in the $n-1$ loci (12) where $A^{1 / n}$ is in turn each nth root of unity except unity. If $A=1$ and (13) is false, we draw no conclusion concerning the location of $z$.

The boundary of the locus (12) consists formally of four algebraic curves. If $r_{1}=0$ these curves are either circles or lemniscates, perhaps degenerate. If $a=b$ each of these curves is either obviously a circle or (12) has the form

$$
|z-a| \leqq B_{1}+\frac{B_{2}}{|z-a|}, \quad B_{1} \geqq 0, B_{2} \geqq 0,
$$

which can be written

$$
\begin{aligned}
{\left[|z-a|-\frac{B_{1}}{2}+\frac{1}{2}\left(B_{1}^{2}+4 B_{2}\right)^{1 / 2}\right] } \\
\cdot\left[|z-a|-\frac{B_{1}}{2}-\frac{1}{2}\left(B_{1}^{2}+4 B_{2}\right)^{1 / 2}\right] \leqq 0 .
\end{aligned}
$$


If $B_{2}=0$, (14) defines a disc; if $B_{2}>0$, the first factor of (15) is automatically positive for all $z$; thus (14) defines a circular disc.

The proof of Theorem 2 is similar to that of Theorem 1 and is left to the reader. However, we remark that the proof continually involves equations of the form

$$
z-\alpha=A^{1 / n}(z-\beta), \quad A^{1 / n} \neq 1 ; \quad z-\alpha=z-\beta .
$$

When $\alpha$ and $\beta$ are known to lie in given discs, the first of equations (16) implies that $z$ lies in a related disc as in (12); but the second equation implies no conclusion concerning the location of $z$ if the given discs containing $\alpha$ and $\beta$ are not disjoint, and is an impossibility ( $z$ does not exist) if those discs are disjoint.

\section{REFERENCES}

1. J. H. Grace, The zeros of a polynomial, Proc. Cambridge Philos. Soc. 11 (1902), 352-357.

2. J. L. Walsh, On the location of the roots of certain types of polynomials, Trans. Amer. Math. Soc. 24 (1922), 163-180.

3. - The location of the zeros of the derivative of a rational function, revisited, Abstract 617-244, Notices Amer. Math. Soc. 8 (1961), 447.

4. - The location of the zeros of the derivative of a rational function, revisited, J. Math. Pures Appl. (to appear).

HARVARD UNIVERSITY 